\title{
Integrated remotely sensed datasets for disaster management
}

\author{
Timothy McCarthy ${ }^{1}$, Ronan Farrell ${ }^{2}$, Andrew Curtis ${ }^{3}$ and A. Stewart Fotheringham ${ }^{1}$ \\ ${ }^{1}$ National Centre for Geocomputation, National University of Ireland, Maynooth, (NUIM) Ireland \\ ${ }^{2}$ Institute of Microelectronics \& Wireless Systems, NUIM, Ireland \\ ${ }^{3}$ Department of Geography, University of Southern California, Los Angles CA 90089-0255
}

\begin{abstract}
Video imagery can be acquired from aerial, terrestrial and marine based platforms and has been exploited for a range of remote sensing applications over the past two decades. Examples include coastal surveys using aerial video, routecorridor infrastructures surveys using vehicle mounted video cameras, aerial surveys over forestry and agriculture, underwater habitat mapping and disaster management. Many of these video systems are based on interlaced, television standards such as North America's NTSC and European SECAM and PAL television systems that are then recorded using various video formats. This technology has recently being employed as a front-line, remote sensing technology for damage assessment post-disaster.

This paper traces the development of spatial video as a remote sensing tool from the early 1980 s to the present day. The background to a new spatial-video research initiative based at National University of Ireland, Maynooth, (NUIM) is described. New improvements are proposed and include; low-cost encoders, easy to use software decoders, timing issues and interoperability. These developments will enable specialists and non-specialists collect, process and integrate these datasets within minimal support. This integrated approach will enable decision makers to access relevant remotely sensed datasets quickly and so, carry out rapid damage assessment during and post-disaster.
\end{abstract}

Keywords: Remote Sensing, GIS, GPS, Spatial Video, Navigation, Mobile Mapping, Interoperability, Mobile Devices

\section{Introduction}

Spatial encoded video can be defined as technologies that enable geospatial information to be associated with motion picture sources and integrated with other geo-databases within a GIS or mapping environment. Figure 1 depicts a spatial encoded video browser comprising video, map and spatial database modules, all of which are integrated within a GIS environment. Spatially encoded video can trace its remote sensing roots back to the 1980s following developments in charged coupled device (CDD) technology [5] and Global Positioning System (GPS), [28]. Various camera configurations were used for a variety of mapping and monitoring tasks [2]. These camera systems were, for the most part, fixed to airborne platforms and acquired multipsectral imagery across visible and near infra-red bands. The video sensors included both specialised progressive scan cameras as well as commercially available VHS and S-VHS camcorders. This imagery was processed post-sortie on the ground using geometric, radiometric algorithms that were developed for satellite remote sensing. Aerial video data was usually integrated with satellite earth observation data since additional spatial information could be gleaned from the resulting data product. The synoptic, georeferenced satellite dataset provided a natural base-map to reference video frames or mosaiced video imagery. This was especially true if there was little if any existing aerial photography or map data available. Conversely, aerial video imagery could be used to ground truth and/ validate the results of remote sensing classification. Aerial videography surveys could be commissioned at relatively short notice and could be directed to the area of interest. The relatively small sensor size and associated optics meant that this remote sensing tool was predominantly used for small area survey or of areas having a linear extent. Examples of the former include small agriculture plots whilst areas with linear extent include; river habitats, coastal-zone and route corridors. 


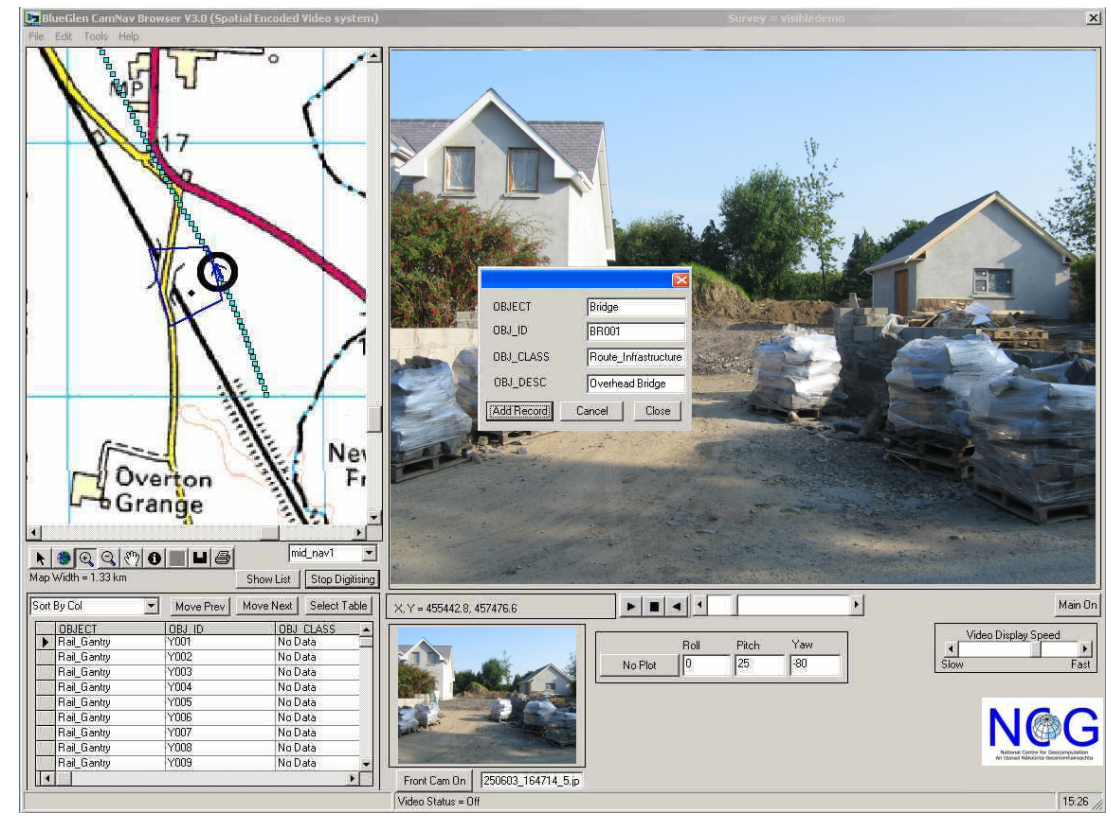

Fig 1. Browser depicting integration of spatial video within a GIS

Various approaches were adopted to minimise problems caused by interlaced, television systems as well as video recording formats [15]. Advances in other data processing areas included multi-frame rectification where very large numbers of overlapping video frames are automatically ortho-rectified [3] and mosaiced [4]. In the early 1990s, the concept of direct georeferencing appeared [10, $11 \& 12]$. This development enabled integration of full positional and orientation information together with image data at the time of acquisition. This innovation allowed imagery to be used for mapping purposes without the need for external ground control points. Ohio State University's Centre for Mapping was one of the first research groups to pioneer the development of ground-based, image-mapping systems for route corridor mapping back in the mid-1990s [6, 7 and 8]. Around this time, easy to use GPS encoders appeared [9]. These encoders enabled satellite GPS position to be encoded onto either the video or audio signal. The GPS data could be decoded post-sortie and so, enabled the video stream to have an associated spatial journey or trail. This enabled field scientists and engineers to explore new opportunities for this relatively cheap, compact, technology. At this time video formats also began to improve for example, interlaced video recording resolution increased from 300 lines (VHS) to over 500 lines (DV), [9].

The present generation of spatially encoded imaging systems are sometimes referred to as mobile mapping systems. Mobile mapping systems include survey grade variants as well as simpler, mapping and monitoring units. The former are based on high-quality progressive-scan cameras whilst the latter are generally associated with less expensive camcorder systems. Present day terrestrial applications of both systems range from 3D urban reconstruction [16], simple streetnavigation [23 and 24], road-survey system using high-quality progressive scan cameras [13] to low-cost, GPS encoded systems employed for mapping shallow-water habitats [14]. The less expensive camcorder system is the subject of this paper and typically GPS is encoded using the vertical line interval (VLI) of the video signal or onto one of the audio channels [9]. These compact systems can be deployed on airborne, terrestrial or onboard marine platforms.

\section{Integrating spatial video with other Remote Sensing datasets for disaster management}

There has been increased attention regarding disasters in recent years, at least in part due to real-time media reporting capabilities. For example, 2008 has already seen devastating cyclones, earthquakes, tornadoes and wildfires. These disasters can broadly be categorized into three areas; natural events such as the Tornadoes [19] that have battered the United States during 2008, technological and terrorist events such as the world trade centre terrorist attack in 2001, [18], and combinations of both a natural phenomena and human agency, such as Hurricane Katrina. The scope or various 
functional elements of disaster management can be described using a 3D matrix, Fig 2. Pre- and post-disaster functions occupy one axis, methodologies employed another axis whilst the third axis describes management structure [1]

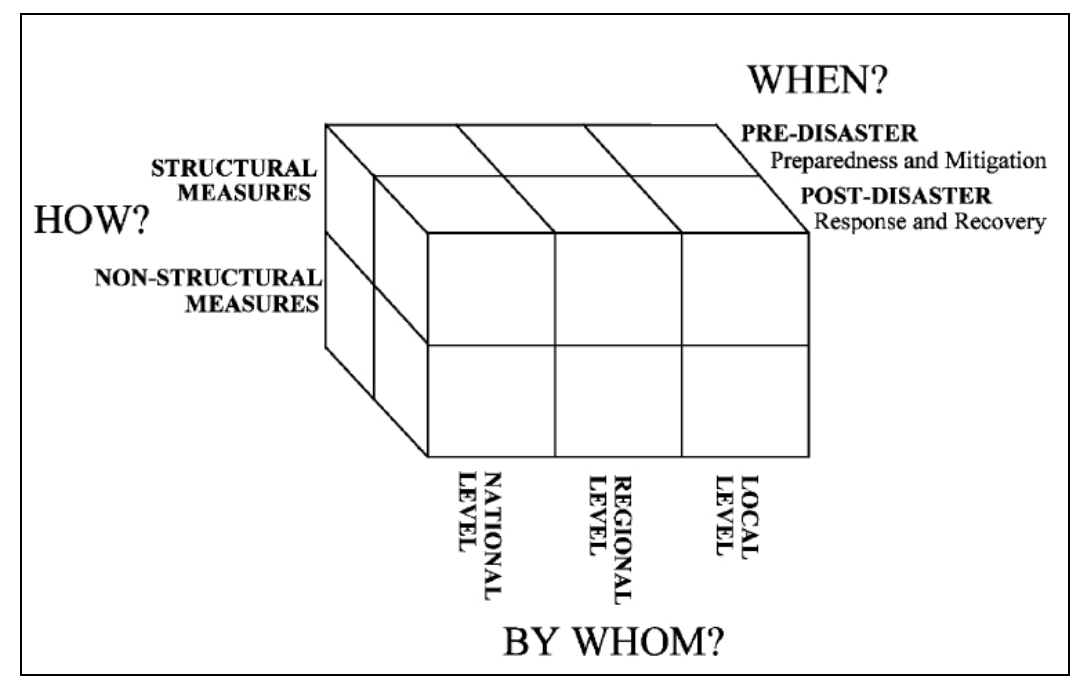

Fig. 2 Functional elements of Disaster Management [1]

Agencies worldwide use remote sensing pre-disaster to map an area of interest or, in some cases, carry out more sophisticated tasks such as risk assessment and mitigation. Post-disaster, remote sensing is used to acquire multithematic data and so, help relevant authorities to respond in an efficient manner. Both satellite and airborne remote sensing can provide multi-sensor, bird's eye view of the event. These datasets enable change detection to be carried out and so provide an indicator of damage caused by the disaster. Mutlispectral data allows more sophisticated attributes such as fire-risk, crop damage or effects of flooding to be measured and classified using multi-temporal datasets. Satellite radar imagery can be acquired and used if cloud cover poses a problem though the nature and scale of disaster can limit the effectiveness of these data[18]. There are additional shortcomings such as resolution, re-visit time and offnadir acquisition geometry for satellite data [18] but these are steadily improving. Other problems include the availability of suitable aircraft equipped with remote sensing sensors close to the disaster. Airborne and satellite data will, by and large, be restricted to a high altitude, synoptic view. This can result in information gaps depending on disaster scenario [17].

Timely, spatial encoded image data captured by non-specialists on the ground or close to the disaster can help fill these gaps [17 \& 19]. These systems are easy to use and usually comprise a camcorder and GPS encoder. The user can walk or drive around the target area and record GPS encoded video. Near real-time spatial tagged information can be used to compliment existing remote sensing data-sources and manage the recovery process. This human-scale spatial video may include information not readily gleaned by over-flying conventional remote sensing platforms. These include spatial image information regarding damaged infrastructure, unstable building structures, evidence of looting, and in the recovery stage, evidence of return and rebuilding, Fig. 3. All of this information contributes to a better understanding of the damage caused as well as on-going risk posed by the disaster. Some of the key findings of a report after the world trade centre terrorist attack were the need for timely, thematic data and also to ensure that spatial data processing was reasonably straight forward [18]. Spatial video has the potential to satisfy both these requirements. Spatial video has been used post-disaster for both hurricanes and tornadoes. In both cases, it was found to be easy to deploy and collected data that resulted in useful spatial information when integrated with other remotely sensed datasets [17 and 19]. 


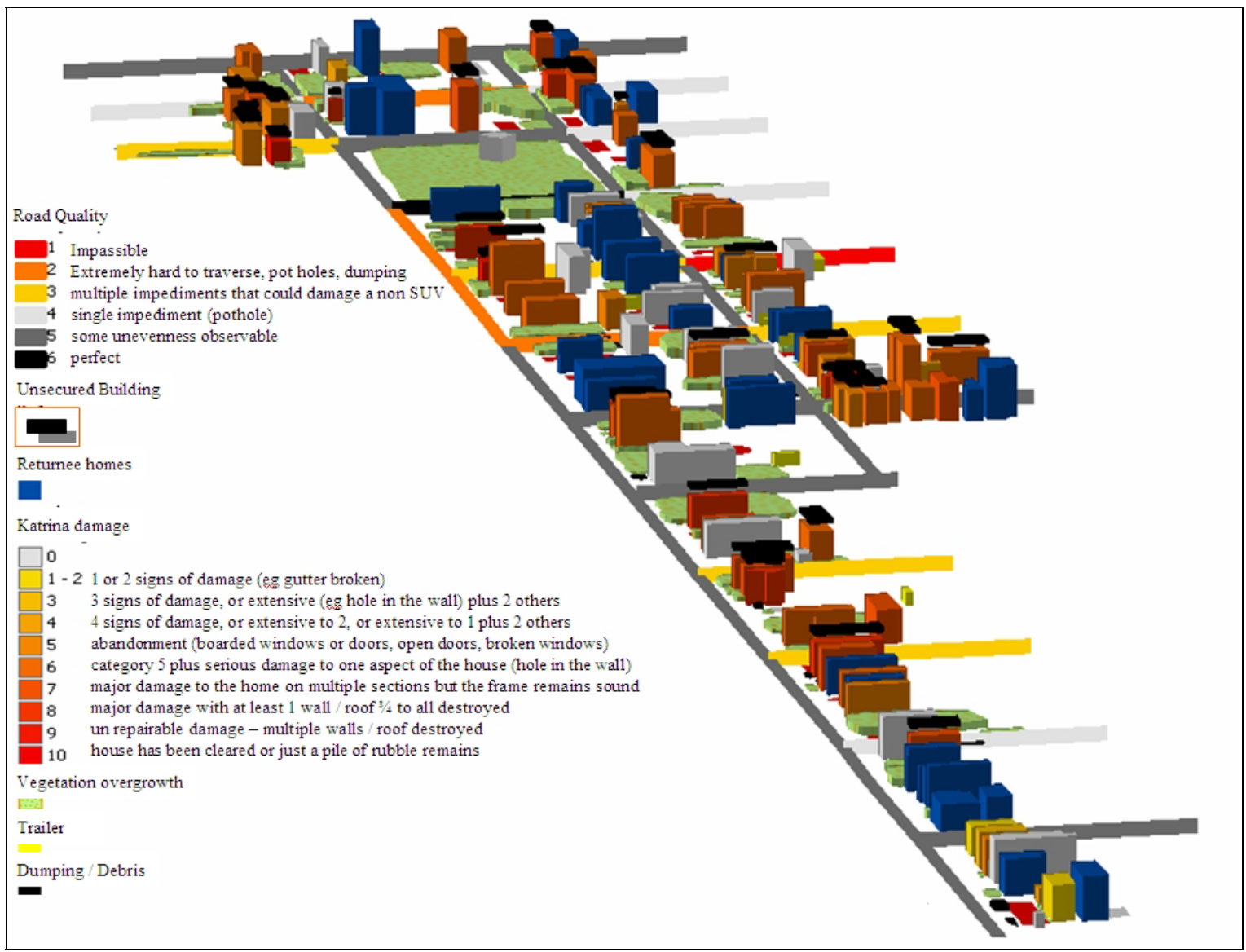

Fig. 3. Damage assessment map at street-level using information collected by spatial-video technology (after A. Curtis 2008)

Present day spatial encoded image mapping systems include those developed commercially as well as those by the research community. Commercial systems include those provided by Navtech Systems [20], Horita [21] and Red Hen Systems [22]. National University of Ireland, Maynooth (NUIM) has developed video mapping technology. This system, based on earlier research [9], enables spatial video to be integrated with a GIS. The user is able to navigate through the spatial video using a browser and can record features of interest for example, building damage and store these events in a database for later retrieval. In some cases, the monoscopic video field of view is calibrated after it is fixed to a survey vehicle, Fig. 4. 


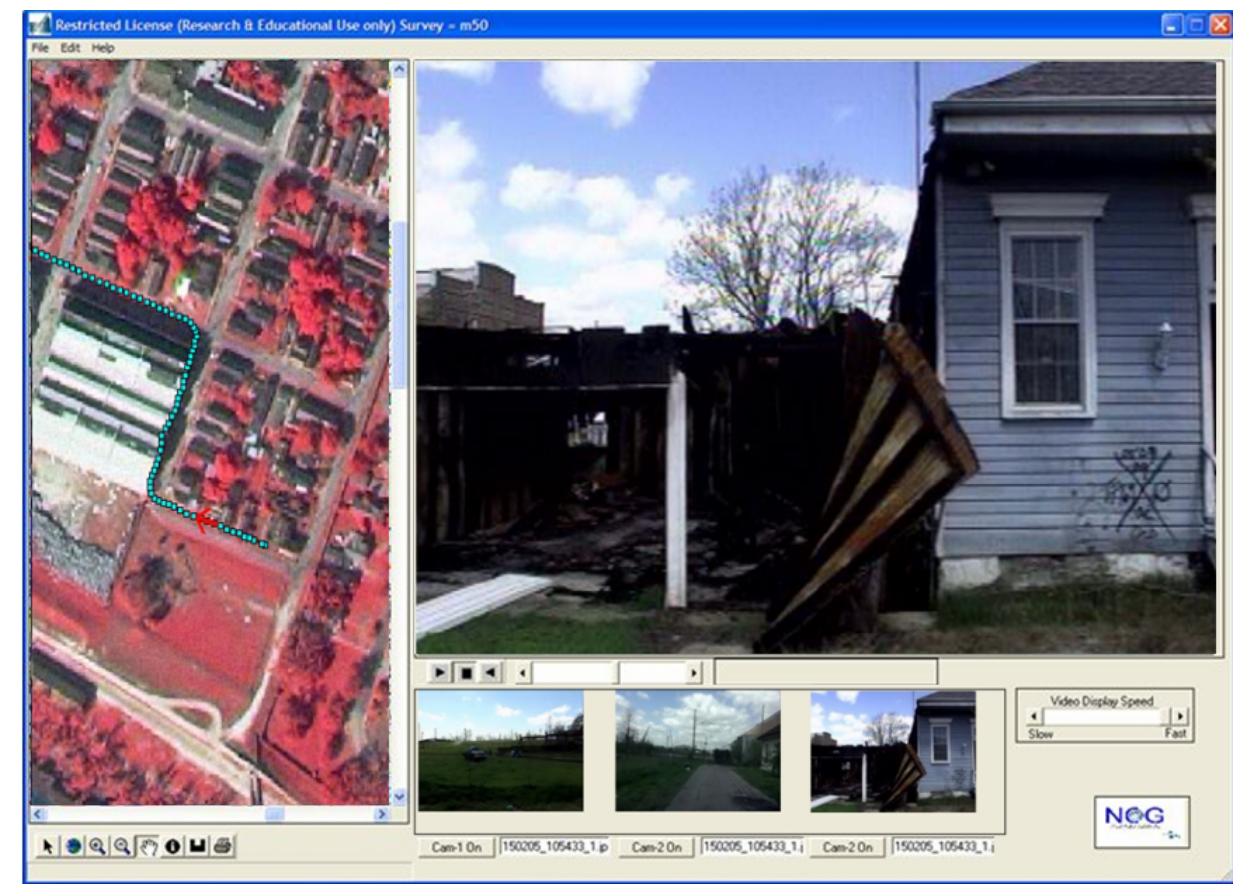

Fig 4. Spatial video technology collected from a vehicle using three camcorders for Post-Katrina New Orleans

This calibration enables the user to extract simple 2D measurements from the resulting spatial video. NUIM has also worked with colleagues at University of Southern California [19] to help test spatial video technology for post-disaster mapping.

\section{Motivation for research}

The NUIM research team found shortcomings in the current system. The timing relationship between GPS position and associated video frame is not well understood for some of these systems. These sensors operate in asynchronous fashion for example $50 \mathrm{~Hz}$ PAL (Field) Video and $1 \mathrm{~Hz}$ GPS. There are latencies between various sensing, computation and recording functions. GPS sensors can have latencies in the order of 100s of milliseconds when acquiring satellite signals, computing position and outputting the solution on RS-232. These latencies can be further affected by the time it takes to encode the GPS and write to AV data-stream [9]. System latency becomes an issue when positional accuracy is important or if the user wishes to synchronise a number of camcorders that may have different fields of view. The advent of new, high definition (HD) video formats such as $720 \mathrm{p}$ and $1080 \mathrm{p}$ will probably encourage potential users to include these sensors for survey grade work. Accurate synchronisation of video and navigation sensors will be critical in ensuring this development.

Typically, these systems use hardware units to encode GPS onto audio-video (AV) camcorders out in the field. Once the user returns to the laboratory or office, the same hardware encoder is switched to decode mode in order to extract and rebuild the relationship between the geographic position and associated video. Encoding GPS onto camcorders will, for the short term at least, involve using a small hardware module. Anybody who is competent in using a camcorder and GPS will be able to encode GPS onto video. Processing this data still poses some problems since the user has to connect cables, configure both software and hardware each time, Fig 5. The decoding step typically demands a reasonable level of technical skill to execute. This introduces inefficiencies as well as associated capital costs in producing spatial information. 


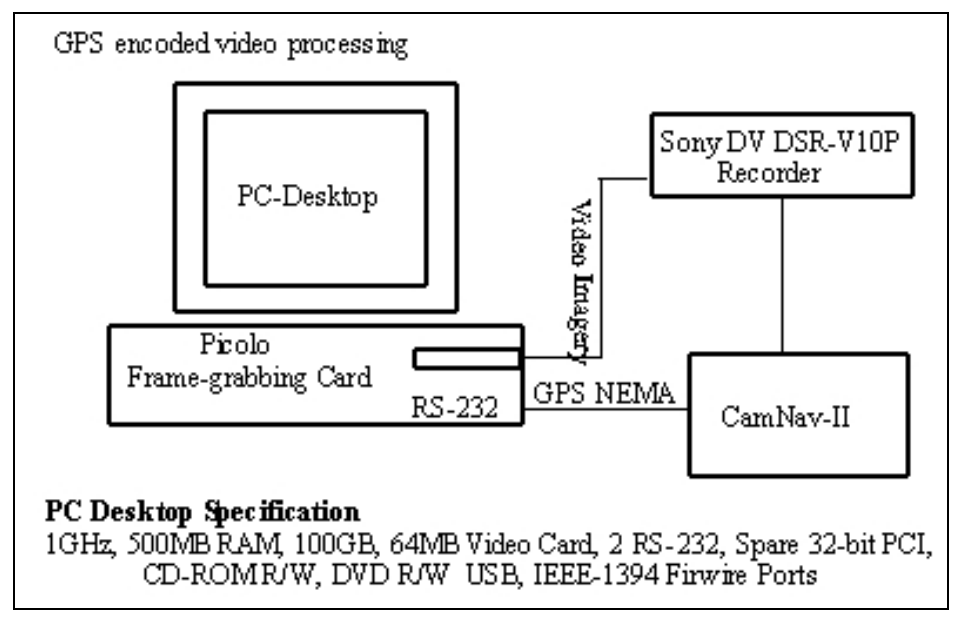

Fig 5. Schematic of decoding components for an older spatial video system

Another less well investigated area concerns seamless integration of spatial data with other remote sensing and spatial databases. This is not a trivial problem since spatial video is composed of two different sensor data-streams. The data is dynamic in nature and can be collected and stored using a myriad of video formats. All of this is further complicated by video protocols, web mapping standards as well as standards for new mobile technologies including location based services (LBS).

\section{Prototype system}

Frame accurate synchronisation, easy data processing and seamless geospatial integration provided the drivers for initiating this research. The research team wished to design and build a prototype spatial video mapping system where both encoding and decoding activities could be investigated. A testbed encoder was required to enable additional navigation sensors to be added and where sensor latency issues could also be examined. Additional orientation sensor modules such as Honeywell's latest micro electro mechanical systems (MEMS) digital compass, HMR3600 $\mu$ POINT ${ }^{\circledR}$ can be easily integrated with the encoder. This new sensor is capable of resolving pitch, roll and yaw to $0.1^{\circ}$ at the $0.5^{\circ}$ accuracy level using integrated gyros and accelerometers, [25]. The sensor can also compensate for transient magnetic disturbances. This type of sensor is important if the user wishes to record the orientation of the video sensor as well as the position. An automated software decoder module was required since processing spatial video using conventional approach can be complicated and this improvement would help make spatial video mapping more accessible to a wider audience. Examining interoperability was the third objective since this would enable integration with other remotely sensed datasets. The current status of our testbed system comprises a hardware GPS encoding component and a software decoding module is described in the following sub-sections.

\subsection{Encoder Module}

The navigation message is encoded onto the left audio channel. A pulse-position encoded amplitude shift keying modulation technique is used to encode the alpha-numerical navigation information, Fig 6 . Each alphanumerical value is stored as a pulse-position modulated signal where the pulse corresponds to a period of time where there is tone in the audible frequency range. The period of time between pulses corresponds to a numerical value. In this manner, a string of alphanumerical data can be encoded, the full signal requiring approximately 0.2 seconds. Each navigation message is preceded by a pre-defined signature pattern alerting the decoding algorithm to identify the timing of the signal and to identify commencement of a valid message. This allows the encoded information to be preserved if the speed at which the video is played is altered. 


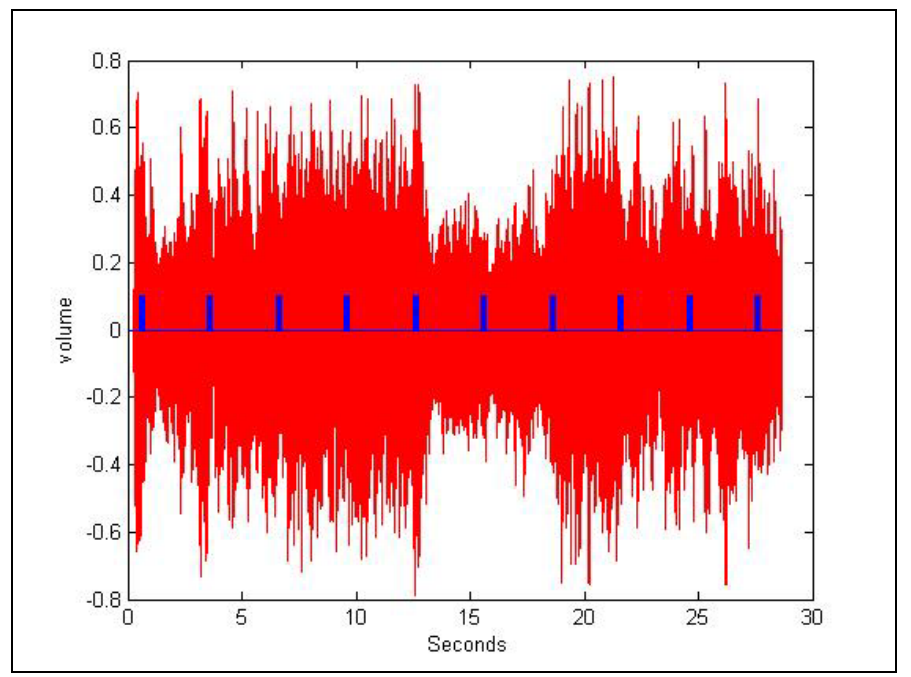

Fig 6. Sample audio waveform depicting simple numerical encoding superimposed on voice recording (NUIM testbed audio encoder)

\subsection{Decoder module}

The user collects spatial encoded video out in the field. This spatial encoded video is then uploaded onto a desktop or laptop as a MPEG movie file. There is no need for the user to connect any cables or configure any decoding system. The video file can be opened by standard windows video players or by the prototype decoding software module. The software decoder module is designed to scan the audio stream searching for navigation messages. Decoding these values commences once valid navigation signature patterns are found. The decoding software is designed to check network connectivity and automatically establish an internet connection if available. The navigation message can be plotted in spatially aware map servers for example Google Maps using their application programming interface (API), [26]. Currently and associated index file is stored once the entire file has been decoded. This enables the entire route to be plotted at the start of the next decoding session and also allows random access to any particular position.

\subsection{Interoperability}

Creating suitable standards and integration will maximise the usefulness of this relatively new spatial data type. Some researchers have examined the standard for description and search of audio and visual content using MPEG-7 [27] whilst some others have developed a prototype storage and query system for encoded video systems [32] based on MPEG-21, the Multimedia Framework, [29]. Open Geospatial Consortium (OGC) have begun working on interoperability issues associated with video data under SensorML and spatial video (GeoVideo) [31]. The research team are examining existing and proposed metadata schemes for storing spatial descriptors as well as optimising interoperability. There are challenges since the intention here is to open this technology to a wider audience and so, be used by specialist and nonspecialist alike. How can a standard attempt ensure that a particular user collects reasonably accurate positional information or indeed suitable spatial imagery. If sensor orientation or geometry information is un-available, how can this same standard store information relating not only observer position but also the geometry of field of view. There are further issues relating to the growing number of video formats [30] and operating environment scenarios such as Internet, mobile devices and desktop PCs. These issues are important to ensure a reasonable level of performance when carrying out spatial query and/or spatial database integration.

\subsection{Timing Issues}

The camcorder was pointed at the test screen of audio signal generator whilst the audio output was plugged directly into the camcorder. The audio signal generator was programmed to pulse various patters over a period of 26 seconds of Timing signals were compared between the video data-stream captured at $25 \mathrm{~Hz}$ and the associated audio track. Audio timing was available at the much higher sampling rate of $32 \mathrm{kHz}$. Each time pulse could be measured at the tenths of a 
millisecond level where the time of each pulse was noted on the rising edge as it passed through the zero. All of these timings were recorded onto a spreadsheet. There were 672 frames at $25 \mathrm{~Hz}$ indicating a total time of $26.88 \mathrm{~s}$. There were 860,175 samples at $32 \mathrm{kHz}$, indicating a total duration of $26.88047 \mathrm{~s}$. The average difference between audio and video time was $0.0546 \mathrm{~s}$ where the pulse on the video is always measured ahead of the same pulse on audio track. Some of this latency can be explained by the audio signal generation software as well as the $40 \mathrm{~ms}$ video frame sampling rate of the video channel. This $0.054 \mathrm{~s}$ latency would introduce a positional error of $1.51 \mathrm{~m}$ if the observer was acquiring spatial video at $100 \mathrm{~km} / \mathrm{hr}$.

\section{Discussion}

Remote sensing has developed from large, complex, specialist systems collecting earth observation data to now include cheaper, non-specialist solutions taking advantage of emerging and converging sensor technology. This technology is capable of recording both spatial and temporal attributes of the target scene in a format that is instantly recognisable to any human being. Its usefulness has been documented across a range of application area including disaster management. One of the fundamental issues restricting access by a wider, global user-base revolves around system complexity. System design should take this into account and ensure ease of use so that it doesn't require a manual to explain operation. The system should be compatible with any high-street camcorder system and as easy to operate as a domestic television. Another hurdle concerns interoperability. A suitable protocol that not only addresses present spatial video scenarios but also future possibilities is required in order to maximise integration not only for Web based mapping systems but also mobile devices. This standard should be able to handle the specialist who wishes to integrate spatial video with other remote sensing datasets in ArcGIS or ERDAS. This same standard should be able to cater for people who wish to upload their spatial video movie whether recording events post-disaster, collecting data for spatial and temporally changing for other urban pursuits (housing foreclosures and crime being an obvious example) or even some holiday trip. These spatial-video blogs could be uploaded and handled as GeoYouTube, a spatial-video variation of the more popular YouTube, [33]. Particular attention needs to be given to the various emerging technologies onboard mobile devices especially those that mirror the underlying components of spatial video. These components include imaging sensors and positioning sensors. Wireless protocols enable real-time data collection as well as geoinformation broadcast to be explored. These enabling technologies bring the fundamental idea of remote sensing directly to the pockets of millions of potentials users. This is where people now become both the collectors and end-users of timely, spatial information relevant to their particular needs.

\section{Conclusions}

This paper traced the roots of spatial video from the 1980s to the present day. The importance of remote sensing in disaster management was investigated. Some of the findings point to a requirement for additional remote sensing data that was human-scale and collected along the ground using simple sensor technology. The motivation behind this research initiative included a desire to examine system complexity, interoperability and timing issues. The current status of the new prototype system was described. This included a new encoding scheme, software decoding architecture, system latency assessment as well as interoperability issues. Some of the main impediments to wider take-up of spatial video are system complexity and interoperability issues. The system needs to be easy to use and interfaces with other systems such as geodatabases should be seamless. Mobile devices such as cell phones now carry multiple chipsets capable of recording video and computing position. All of the issues tackled by this research initiative will have direct relevance to enabling these new mass-market sensors carry out remote sensing tasks. The potential of wireless capability not only means that this data could be collected in real time but that the user could carry out spatial queries for scenarios of immediate interest to them. These new scenarios raise both opportunities as well as research challenges.

\section{ACKNOWLEDGEMENTS}

Research presented in this paper was funded by a Strategic Research Cluster grant (07/SRC/I1168) by Science Foundation Ireland under the National Development Plan. The authors gratefully acknowledge this support. 


\section{REFERENCES}

[1] Montoya L. "Geo-data acquisition through mobile GIS and digital video: an urban disaster management perspective" Environmental Modelling \& Software, Volume 18, Issue 10, Pages 869-876. 2003.

[2] King D.J. "Airborne mutispectral digital cameras and video sensors. A critical review of system design and applications". Canadian Journal of Remote Sensing. Vol 21 (3), pp 245 - 273. 1995.

[3] Holm M "New technology for making huge image mosaic maps and digital elevation models using parallel computing". CSC Computing. CSC News, Vol 10, No 1. pp 21 - 23. 1998.

[4] Linden DS, Hoffer RM, Pywell HR and Bobbe TI "Automated digital mosaicking of airborne videography". Aerial Video workshop. Third international airborne remote sensing conference and exhibition. 7th-10th July. Copenhagen, Denmark. 1995.

[5] Meisner, D.E. "Fundamentals of Airborne video Remote Sensing. Remote Sensing of Environment". 19 pp 63 - 79. 1986.

[6] Blaho, G., and Toth, C. "Field Experiences with a Fully Digital Mobile Stereo Image Acquisition System". Proc. ASPRS-ACSM Annual Convention, Vol. 2, pp. 97-104, 1995.

[7] Bossler, J. D., and Toth, C. "Accuracies Obtained by the GPSVan"M", Proceedings, IS/LIS'95, Vol. 1., pp. 70-77, 1995.

[8] Bossler, J., and Toth, C. "Feature Collection by the GPSVan ${ }^{\mathrm{TM}}$, Analog Video or Stereo Digital Video?", Proceedings, ASPRS-ACSM Annual Convention, Vol. 2, pp. 108-115, 1996.

[9] McCarthy T. Integration of aerial video with a GIS. PhD Thesis. University of London (Birkbeck). 1999.

[10] Toth C and Grejner-Brzezinska D. "Performance analysis of the airborne integrated mapping system (AIMS)". International Archives of Photogrammetry and Remote Sensing, 32(2): pp 320-326.1998.

[11] Mostafa M and Schwarz K. "A multisensor system for airborne image capture and georeferencing". PERS. December 200 pp 1417 - 1423. 2000.

[12] McCarthy T., Fotheringham A.S. and O'Riain G. "Compact Airborne Image Mapping System (CAIMS)". The International Archives of the Photogrammetry, Remote Sensing and Spatial Information Sciences/, Padua, Italy, Vol.

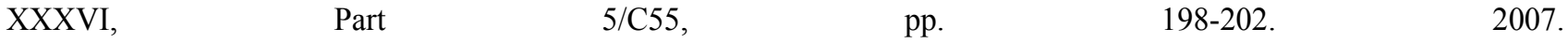

[13] McCarthy T., Charlton M., Fotheringham A.S. and O'Malley V. "Integration of LiDAR and Stereoscopic Imagery for Automated Route Corridor Inventory". The International Archives of the Photogrammetry, Remote Sensing and Spatial Information Sciences/, Padua, Italy, Vol. XXXVI, Part 5/C55, pp. 203-209. 2007.

[14] Lirman D. and Deangelo G. "Geospatial video monitoring of benthic habitats using shallow-water positioning system”. Oceans 2007. pp 1-5. 2008.

[15] Neale CMU, Kuiper J, Tarbet KL and Qiu X. "Image Enhancement and Processing Automation Routines for Digital Multispectral Video Imagery". Proceedings of the 14th Biennial Workshop on colour aerial videography and videography for resource monitoring. Logan, Utah, May 25th - 29th. 1993. 
[16] Akbarzadeh A., Frahm J.-M.,Mordohai P., Clipp B., Engels C., Gallup D., Merrell P., Phelps M., Sinha S., Talton B., Wang L., Yang Q., Stewenius H., Yang R., Welch G., Towles H., Nister D., and Pollefeys M. "Towards Urban 3D Reconstruction from Video", 3dpvt, pp. 1-8, Third International Symposium on 3D Data Processing, Visualization, and Transmission (3DPVT'06). 2006.

[17] Curtis A, Mills J. W., Kennedy B., Fotheringham A. S. and McCarthy T. "Understanding the geography of posttraumatic stress: An academic justification for using a spatial video acquisition system in the response to Hurricane Katrina". Journal of Contingenices \& Crisis Management. Volume 15. No 4. pp 209-219. 2007

[18] Huyck C. and Adams B. "Emergency response in the wake of the World Trade Centre Attack : The Remote Sensing Perspective". Engineering and organiszational issues related to the world trade centre terrorist attack. Vol 3. pp 45. 2002.

[19] Mills, Cutis, Fagan and Core "The Spatial Video Acquisition System as an Approach to Capturing Damage and Recovery Data After a Disaster: A Case Study from the Super Tuesday Tornadoes". http://www.colorado.edu/hazards/research/qr/qrpubs.html. 2008.

[20] Navtech Systems. CamNav System. www.navtechsyetems.co.uk. 2008.

[21] Horita. Horita GPS encoding device. www.horita.com. 2008.

[22] Red Hen Systems. Red Hen Systems MediaMapper. www.redhensystems.com. 2008.

[23] Kimber D., Foote J., and Lertsithichai S. "FlyAbout: Spatially Indexed Panoramic Video", in Proc. ACM Multimedia 2001, Ottawa, CA. 2001.

[24] Google Street Maps. Interactive Streetview. http://maps.google.com/help/maps/streetview/. 2008.

[25] Honneywell Orientation Sensor (2008). Honeywell MEMS HMR3600 $\mu$ POINT®. http://www.ssec.honeywell.com/magnetic/products.html

[26] Google Maps API. Application Programmer Interface, Goolge Maps. http://code.google.com/apis/maps/. 2008.

[27] Chang S., Sikora T., and Puri A. "Overview of the MPEG-7 Standard". IEEE TRANSACTIONS ON CIRCUITS AND SYSTEMS FOR VIDEO TECHNOLOGY, VOL. 11, NO. 6, pp 688 - 695. 2001.

[28] Parkinson, B.W. Gilbert, S.W. "NAVSTAR: Global positioning system-Ten years later". Proceedings of the IEEE. Volume: 71, Issue: 10. 2005.

pp 1177- 1186

[29] MPEG-21 Multimedia Framework Standard (2008) http://www.chiariglione.org/mpeg/standards/mpeg-21/mpeg21.htm

[30] Video File Formats. http://www.fileinfo.net/filetypes/video. 2008.

[31] Open Geospatial Consortium (OGC). http://www.opengeospatial.org/. 2008.

[32] O’Connor N.E., Duffy T., Ferguson P., Gurrin C., Lee H., Sadlier D.A., Smeaton A.F. and Zhang K. “A Contentbased Retrieval System for UAV-like Video and Associated Metadata”. Airborne Intelligence, Surveillance,

Reconnaissance (ISR) Systems and Applications V. Proc. of SPIE Vol. 6946, 69460. 2008.

[33] YouTube http://www.youtube.com/. 2008. 